\title{
Roles and applications of 3D printing in gynecology: a scoping review
}

Carly Cooke, Teresa Flaxman, Lindsey Sikora, Sony Sukhbir Singh

\section{Citation}

Cooke CM, Flaxman TE, Sikora L, Singh SS. Roles and applications of 3D printing in gynecology: a scoping review

\section{Review Question}

- What are the roles and applications of 3D printing in gynecology?

- What is the feasibility and impact of 3D printing on surgical outcomes in gynecologic surgery?

\section{Searches}

- The following databases will be searched: Medline, Embase, CENTRAL, Scopus

- In addition, supplementary searching will include a cited reference search and gray literature search to include conference abstracts from the following journals, Journal of Obstetrics and Gynecology of Canada, Journal of Minimally Invasive Gynecology, and Obstetrics and Gynecology

Types of study to be included

- Inclusion criteria:

- Gynecologic surgical context

- English

- All types of studies, with inclusion of conference abstracts

- Studies up to May 2021

- Studies where 3D printed models were used as patient-specific devices (i.e patient specific pessaries, vaginal dilators)

- Studies where 3D printed models were used as moulds for producing generic devices (i.e for teaching)

- Exclusion criteria:

- Studies involving 3D imaging alone, without 3D model production

- Studies where 3D printing is used for bioprinting, scaffolding, tissue engineering

- Studies where 3D printing was used in a purely obstetrical context (i.e for fetal imaging, or for the purpose of investigating fetal pathology)

\section{Condition or domain being studied}

Segmentation software has allowed 2-dimensional ultrasound and Magnetic Resonance (MR) images, which are commonly ordered as part of the investigation of gynecologic pathologies, to be converted to 3D printed models. Hence, 3D printed models of patientspecific female pelvic anatomy and gynecologic pathology are now being produced. Some of the applications for 3D printing which have been identified in the literature for uses in other surgical specialities, including surgical planning and trainee and patient 
education, have been investigated in the context of gynecology. A broad range of clinically meaningful applications of 3D printing in gynecology have been identified, but have yet to be summarized in a succinct way. Our objective is to review the literature on $3 \mathrm{D}$ printing in gynecology to 1) outline the roles, applications of 3D printing in gynecology and 2) determine the feasibility and impact of 3D printing on surgical outcomes in gynecologic surgery. This will help to establish the overall clinical relevance of $3 \mathrm{D}$ printing in gynecology.

Participants/population

Obstetrician/gynecologists of all ages and all levels of training including staff, fellows, residents and medical students training in Obstetrics and Gynecology. Physicians from other specialties and gynecology patients will also be included.

Intervention(s), exposure(s)

Utilization of 3D printed models

$\underline{\text { Comparator/control }}$

$\mathrm{N} / \mathrm{A}$

Main Outcome

Description of the clinical applications of 3D printing in Gynecology

Additional Outcomes

- Measures of feasibility (3D printing costs, production time)

- Surgical outcomes in gynecology (operative time, estimated blood loss, complication rate)

\section{Data extraction (selection and coding)}

Studies will be screened and assessed for eligibility by two independent reviewers (CC, TF). Reviewers will then extract data from studies selected for inclusion using a preestablished data extraction form. Disagreements between reviewers regarding study screening, eligibility, and data extraction will be settled through discussion and consensus between the reviewers.

Strategy for data synthesis

We plan to use a descriptive approach for data synthesis, gathering and summarizing qualitative data and narrative discussions regarding the utility of 3D printing in gynecology. We will also assess quantitative data when available, which may include costs, production time, surgical outcomes.

Analysis of subgroups or subsets

None planned

Contact details for further information

Carly Cooke

carcooke@ohri.ca 
Organisational affiliation of the review

Department of Obstetrics and Gynecology, University of Ottawa, Ottawa, ON;

Department of Clinical Epidemiology, Ottawa Hospital Research Institute, Ottawa, ON

Health Sciences Library, University of Ottawa, Ottawa, Ontario, Canada

Review of team members and their affiliations

Carly Cooke: Department of Obstetrics and Gynecology, University of Ottawa, Ottawa, ON

Teresa Flaxman: Department of Obstetrics and Gynecology, University of Ottawa, Ottawa, ON; Department of Clinical Epidemiology, Ottawa Hospital Research Institute, Ottawa, ON

Lindsey Sikora: Health Sciences Library, University of Ottawa, Ottawa, Ontario, Canada Sony Singh: Department of Obstetrics and Gynecology, University of Ottawa, Ottawa, ON; Department of Clinical Epidemiology, Ottawa Hospital Research Institute, Ottawa, ON

Type and method of review

Scoping review

Anticipated or actual start date

12 May 2021

Anticipated completion date

01 July 2021

Funding sources/sponsors

None

Conflicts of interest

None known

Language

English

Country

Canada

Stage of review

Review ongoing

Subject index terms

- 3D printing; 3D imaging; 3D Model; 3D Anatomy; 3D Digital; 3D rendering; Three dimensional

- Gynecology; ObGyn; Uterus; Vagina; Ovaries; Cervix; Fallopian tubes; Bladder; Urethra; Ureter; Pelvic floor 
Stage of review at time of this submission

\begin{tabular}{|l|l|l|}
\hline Stage & Started & Completed \\
\hline Preliminary searches & Yes & Yes \\
\hline $\begin{array}{l}\text { Piloting of the study selection process } \\
\begin{array}{l}\text { Formal screen of search results against eligibility } \\
\text { criteria }\end{array}\end{array}$ & Yes & No \\
\hline Data extraction & No & No \\
\hline Data analysis & No & No \\
\hline
\end{tabular}

